\title{
Decomposability for division algebras of exponent two and associated forms
}

\author{
Karim Johannes Becher
}

\begin{abstract}
This article investigates the structure of quadratic forms and of division algebras of exponent two over fields of characteristic different from two with the property that the third power of the fundamental ideal in the associated Witt ring is torsion free.
\end{abstract}

Keywords Central simple algebra · Merkurjev's Theorem - Quaternion algebra - Quadratic form - Clifford invariant . Witt index · Height $\cdot$ Real field $\cdot$ Stability index · Virtual cohomological dimension . Field invariants

Mathematics Subject Classification (2000) 11E04 - 11E10 - 11E81 - 12D15 - 12G10 . $16 \mathrm{~K} 50 \cdot 16 \mathrm{~S} 35$

\section{Introduction}

Examples of central simple algebras of exponent 2 and of large index can be obtained by taking tensor products of quaternion algebras. The first essentially different examples have been given by Amitsur et al. [1]. They showed that, over certain ground fields, there exist (central) division algebras of exponent 2 and of large index which are not decomposable into a tensor product of two proper subalgebras. However, the problem to decide whether decomposition is possible for division algebras of exponent 2 over a particular field is still interesting.

Over a field $F$ of characteristic different from 2, Merkurjev's Theorem [17] yields a good description of the 2-torsion part of the Brauer group of $F$ : any central simple algebra of exponent 2 over $F$ is Brauer equivalent to a tensor product of quaternion algebras. This, however, says only little about the structure of a central simple algebra of exponent 2 itself, in particular, it does not say whether the algebra is isomorphic - not only Brauer equivalent- to a tensor product of quaternion algebras.

\section{K. J. Becher (囚)}

Fachbereich Mathematik und Statistik, D203, Universität Konstanz, 78457 Konstanz, Germany

e-mail: becher@maths.ucd.ie 
Merkurjev actually noticed that division algebras of exponent 2 over $F$ decompose into tensor products of quaternion algebras provided that the ground field $F$ is of characteristic different from 2 and has cohomological 2-dimension $c d_{2}(F)=2$; the argument is explained by Kahn [11]. This observation generalizes a phenomenon known for local fields, totally imaginary number fields, and function fields of surfaces over an algebraically closed field: over those fields, any division algebra of exponent 2 is in fact itself a quaternion algebra. On the other hand, there exist fields $F$ with $c d_{2}(F)=2$ and with a division algebra over $F$ of exponent 2 and of large index; this is a consequence of another famous result due to Merkurjev, concerning the $u$-invariant of a field (cf. [18]).

In view of the hypothesis on the cohomological 2-dimension, Merkurjev's decomposability statement mentioned above can only apply to nonreal fields. The main objective of this article is to investigate thoroughly the argument in [11] and to extend the method and the result as far as possible, especially to cover real fields as well. In (6.4) we show that, if $c d_{2}(F(\sqrt{-1})) \leq 2$, then any division algebra of exponent 2 over $F$ which splits over every real closure of $F$ is isomorphic to a product of quaternion algebras; moreover, the assumption on the behaviour of $D$ over the real closures can be omitted if every algebraic extension of $F$ is an $E D$-field.

The restriction of the problem of decomposability to the case of algebras of exponent 2 made in this article is imposed by the method, which depends intrinsically on the use of quadratic forms and thus only applies to this case. The bridge between the realm of central simple algebras of exponent 2 and the realm of quadratic forms is established by the Clifford invariant. A significant part of this article is devoted to the study of quadratic forms over fields satisfying the condition that the third power of the fundamental ideal in the associated Witt ring is torsion free. Over fields with this property, quadratic forms are completely classified by their classical invariants (cf. [7]), so at first glance they may seem to be perfectly understood. That this is an illusion is highlighted by some striking open questions concerning quadratic forms in this situation. Some of those problems will be formulated here and several new results are obtained.

All fields considered in this article are assumed to have characteristic different from 2. Analogues in characteristic 2 to most of the results are likely to exist, but a special machinery will be necessary to deal with this case. Several results obtained in this article are already known in the case where the field is nonreal, so a special focus lies on real fields.

\section{Preliminaries}

This article is based on three well-established theories which are related to each other, namely the theory of central simple algebras, the theory of real fields and their orderings, and quadratic form theory. In this section we fix the terminology and collect some basic facts in these areas, and we obtain some preliminary results.

Let $F$ always denote a field of characteristic different from 2 .

Central simple algebras

General references for the theory of central simple algebras are [5, 20], but also [24, Chap. 8] covers what will be used here.

An $F$-algebra $A$ is said to be central simple if $A$ is finite-dimensional over $F$, if the center of $A$ is $F$, and if $A$ has no nontrivial two-sided ideals. By Wedderburn's Theorem, any such algebra is isomorphic to a matrix algebra $M_{n}(D)$ for some integer $n \geq 1$ and a 
finite-dimensional central division algebra $D$ over $F$, both uniquely determined by $A$. Central simple algebras without zero-divisors are referred to as division algebras, for short.

Two central simple algebras $A$ and $B$ over $F$ are Brauer equivalent if $A \cong M_{m}(D)$ and $B \cong M_{n}(D)$ for certain $m, n \geq 1$ and the same division algebra $D$ over $F$. The Brauer group of $F$, denoted by $\operatorname{Br}(F)$, is the set of classes of central simple $F$-algebras modulo Brauer equivalence, endowed with the operation given by the tensor product of algebras. This group operation is commutative and it will be denoted here with a plus sign. The neutral element of $\operatorname{Br}(F)$ is the class consisting of the $F$-algebras which are split, that is, isomorphic to $M_{n}(F)$ for some $n \geq 1$.

Let $A$ be a central simple algebra over $F$. Then $\sqrt{\operatorname{dim}_{F}(A)}$ is an integer, called the degree of $A$ and denoted by $\operatorname{deg}(A)$. If $D$ is the division algebra over $F$ such that $A \cong M_{n}(D)$ for some $n \geq 1$, then the degree of $D$ is called the index of $A$ and denoted by ind $(A)$; we then have $\operatorname{deg}(A)=n \cdot \operatorname{ind}(A)$. In particular, $A$ is a division algebra if and only if $\operatorname{ind}(A)=\operatorname{deg}(A)$. One denotes by $\exp (A)$ the order of the class $[A]$ in $\operatorname{Br}(F)$. Since $A^{\otimes \operatorname{ind}(A)}$ is always split, $\exp (A)$ is finite and divides $\operatorname{ind}(A)$. It is further known that $\exp (A)$ and $\operatorname{ind}(A)$ have the same prime factors. For a field extension $K / F$ we denote by $A_{K}$ the central simple $K$ algebra $A \otimes_{F} K$. The index ind $(A)$ is equal to the lowest degree in which there exists a finite separable extension $K / F$ such that $A_{K}$ is split.

We say that $A$ is decomposable if $A \cong B \otimes_{F} C$ for two central simple algebras $B$ and $C$ over $F$, both not isomorphic to $F$; otherwise $A$ is indecomposable. If $A$ is indecomposable, then it is a division algebra; in fact, $A \cong M_{n}(D) \cong D \otimes_{F} M_{n}(F)$ gives a nontrivial decomposition as soon as $n>1$. In turn, any division algebra $D$ over $F$ with ind $(D)=\exp (D)=q$ is indecomposable provided that $q$ is a prime power.

As the exponent of any central simple algebra is finite, $\operatorname{Br}(F)$ is a torsion group. We denote by $\mathrm{Br}_{2}(F)$ the 2-torsion part of $\operatorname{Br}(F)$, consisting of the classes of central simple algebras of exponent 1 and 2.

A quaternion algebra is a central simple algebra of degree 2 . Note that a quaternion algebra over $F$ is either a division algebra, whence of index and exponent 2, or it is split and then isomorphic to $M_{2}(F)$. Since $F$ is of characteristic different from 2, any quaternion algebra $Q$ over $F$ has an $F$-basis $(1, i, j, k)$ where $i^{2}, j^{2} \in F^{\times}$and $k=i j=-j i$. Moreover, the algebra $Q$ is determined up to isomorphism by the elements $a=i^{2}$ and $b=j^{2}$ in $F^{\times}$. In reverse, given $a, b \in F^{\times}$there exists a quaternion algebra over $F$ with a basis $(1, i, j, k)$ as above where $i^{2}=a$ and $j^{2}=b$. This quaternion algebra over $F$ is denoted by $(a, b)_{F}$.

By Merkurjev's Theorem [17], every class in $\mathrm{Br}_{2}(F)$ is given by a tensor product $Q_{1} \otimes_{F}$ $\cdots \otimes_{F} Q_{r}$ where $Q_{1}, \ldots, Q_{r}$ are quaternion algebras over $F$. Given a division algebra $D$ of exponent 2 over $F$ it is therefore natural to ask whether $D$ is isomorphic to a tensor product of quaternion algebras.

Albert showed that any central simple algebra of exponent 2 and degree 4 is decomposable into a product of two quaternion algebras. Such algebras are called biquaternion algebras. This is actually the only exception to the following phenomenon (cf. [10]): for any prime $p$ and any integers $f \geq e \geq 1$ with $(p, e, f) \neq(2,1,2)$, there exists a field $F$ together with an indecomposable division algebra $D$ over $F$ such that $\exp (D)=p^{e}$ and $\operatorname{ind}(D)=p^{f}$.

\section{Real fields}

For details on the theory of real fields the reader is referred to [12,21].

The set of nonzero sums of squares in the field $F$ is denoted by $\sum F^{2}$. By definition the field $F$ is nonreal if $-1 \in \sum F^{2}$, otherwise $F$ is real. 
Recall that $\operatorname{char}(F) \neq 2$. Let $X(F)$ denote the set of orderings of $F$. Artin-Schreier Theory yields the equality $\bigcap X(F)=\sum F^{2} \cup\{0\}$. In particular, the field $F$ is real if and only if it has an ordering. For any ordering $P$ of $F$ we denote by $F_{P}$ the real closure of $F$ at $P$, which is the smallest real closed field containing $F$ to which the ordering $P$ extends, uniquely determined by $P$ up to unique $F$-linear order preserving isomorphism.

The set of orderings $X(F)$ is endowed with the topology generated by the sets

$$
H\left(a_{1}, \ldots, a_{n}\right)=\left\{P \in X(F) \mid a_{1}, \ldots, a_{n} \in P\right\}
$$

where $n \geq 0$ and $a_{1}, \ldots, a_{n} \in F^{\times}$. With this topology, $X(F)$ is called the space of orderings of $F$. It is compact and totally disconnected (cf. [21, (6.5)]). In particular, any open set in $X(F)$ is also closed and vice-versa. The sets of the form $H\left(a_{1}, \ldots, a_{n}\right)$ in $X(F)$ are called basic clopen sets.

If there is an integer $s \geq 0$ such that any basic clopen set of $X(F)$ can be written as $H\left(a_{1}, \ldots, a_{n}\right)$ with $a_{1}, \ldots, a_{n} \in F^{\times}$and $n \leq s$, then the least $s \geq 0$ with this property is called the stability index of $F$ and denoted by $\operatorname{st}(F)$; if no such integer exists, then $\operatorname{st}(F)=\infty$. Note that $s t(F)=0$ means that $F$ is either nonreal or uniquely ordered. $A$ field $F$ is said to have the Strong Approximation Property (abbreviated SAP) if any open set in $X(F)$ is a basic clopen set. There are many equivalent characterizations for this special situation (cf. [21, Sect. 9]); in particular a field $F$ has $S A P$ if and only if $s t(F) \leq 1$. In the sequel we refer to such fields as SAP-fields, for short.

A preordering of $F$ is a subset $T \subsetneq F$ which contains $F^{\times 2} \cup\{0\}$ and is closed under addition and multiplication. If $T$ is a preordering of $F$, then $F$ is a real field and $T^{\times}=T \backslash\{0\}$ is a subgroup of $F^{\times}$containing $\sum F^{2}$. If $F$ is real then $\sum F^{2} \cup\{0\}$ is the smallest preordering of $F$. Given a preordering $T$ on $F$, we write $X_{T}(F)=\{P \in X(F) \mid T \subset P\}$ and obtain that $\bigcap X_{T}(F)=T$.

Quadratic forms

The standard references for quadratic form theory over fields are [14,24]. When we speak about quadratic forms we always assume them to be regular, and we often call them just forms.

Since $F$ is of characteristic different from 2, every quadratic form over $F$ can be diagonalized. Given $a_{1}, \ldots, a_{n} \in F^{\times}$, one denotes by $\left\langle a_{1}, \ldots, a_{n}\right\rangle$ the $n$-dimensional quadratic form $a_{1} X_{1}^{2}+\cdots+a_{n} X_{n}^{2}$ over $F$. If $\varphi_{1}$ and $\varphi_{2}$ are quadratic forms over $F$ we denote by $\varphi_{1} \perp \varphi_{2}$ their orthogonal sum and by $\varphi_{1} \otimes \varphi_{2}$ their product; we further write $\varphi_{1} \cong \varphi_{2}$ to indicate that $\varphi_{1}$ and $\varphi_{2}$ are isometric. Given $m \in \mathbb{N}$ we write $m \times \varphi$ for the $m$-fold orthogonal $\operatorname{sum} \varphi \perp \cdots \perp \varphi$ of a form $\varphi$ over $F$.

A quadratic form over $F$ is said to be isotropic if it represents zero non-trivially, otherwise anisotropic. The quadratic form $\langle 1,-1\rangle$ is also denoted by $\mathbb{H}$ and called the hyperbolic plane. It is the smallest quadratic form over $F$ which is isotropic. A quadratic form over $F$ is said to be hyperbolic, if it is isometric to $i \times \mathbb{H}$ for some $i \in \mathbb{N}$.

Let $\varphi$ be a quadratic form over $F$. Then $\varphi$ has a Witt decomposition

$$
\varphi=\varphi_{a n} \perp i \times \mathbb{H}
$$

where $\varphi_{a n}$ is anisotropic and $i \in \mathbb{N}$. Here, $i$ and $\varphi_{a n}$ are uniquely determined by $\varphi$. The integer $i(\varphi)=i$ is called the Witt index of $\varphi$ and $\varphi_{a n}$ is called the anisotropic part of $\varphi$. Two quadratic forms over $F$ are said to be Witt equivalent if their anisotropic parts are isometric. We denote by diman $(\varphi)$ the dimension of the anisotropic part of $\varphi$ and call this the anisotropic dimension of $\varphi$; in difference to the ordinary dimension, $\operatorname{diman}(\varphi)$ depends 
only on the Witt equivalence class of $\varphi$. We denote by $d_{ \pm}(\varphi)$ the discriminant in $F^{\times} / F^{\times 2}$ of $\varphi$. A six-dimensional form of trivial discriminant is called an Albert form. We write $\Delta_{F}(\varphi)$ for the set of non-zero elements of $F$ which are represented by $\varphi$. The form $\varphi$ is said to be universal if $\Delta_{F}(\varphi)=F^{\times}$. Note that any isotropic form is universal. Given $a \in F^{\times}$one writes $a \varphi=\langle a\rangle \otimes \varphi$. A form $\psi$ over $F$ is similar to $\varphi$ if $\psi \cong a \varphi$ for some $a \in F^{\times}$. The form $\varphi$ is said to be multiplicative if $\varphi$ is hyperbolic or if it is anisotropic and satisfies $a \varphi \cong \varphi$ for every $a \in \Delta_{F}(\varphi)$. Given $a_{1}, \ldots, a_{n} \in F^{\times}$, the $n$-fold Pfister form $\left\langle 1,-a_{1}\right\rangle \otimes \cdots \otimes\left\langle 1,-a_{n}\right\rangle$ is denoted by $\left\langle\left\langle a_{1}, \ldots, a_{n}\right\rangle\right\rangle$. Pfister forms are multiplicative.

We denote by $W F$ the Witt ring of $F$, by $I F$ its fundamental ideal, and by $W_{t} F$ the torsion part of $W F$. If $F$ is nonreal then $W F$ is a torsion group. If in turn $F$ is real then $W_{t} F$ is a proper ideal of $W F$, contained in $I F$. For $n \in \mathbb{N}$, we write $I^{n} F=(I F)^{n}$ and $I_{t}^{n} F=W_{t} F \cap I^{n} F$. We will often have to deal with the condition that $I_{t}^{n} F=0$, i.e. that $I^{n} F$ is torsion free, for a certain $n \geq 1$.

A quadratic form $\varphi$ over $F$ is said to be weakly isotropic (resp.torsion), if there exists $n \geq 1$ such that $n \times \varphi$ is isotropic (resp. hyperbolic). Note that $\varphi$ is torsion if and only if its class in $W F$ is a torsion element. Any nontrivial torsion form is weakly isotropic. A form is said to be strongly anisotropic if it is not weakly isotropic. Over a nonreal field every quadratic form is torsion.

Lemma 2.1 Assume that $I_{t}^{n+1} F=0$ and let $\varphi \in I^{n} F$. Then

(a) For every $a \in \sum F^{2}$ one has $a \varphi \cong \varphi$.

(b) If $\varphi$ is torsion, then $a \varphi \cong \varphi$ for every $a \in F^{\times}$and $\varphi$ is universal.

Proof Let $a \in F^{\times}$. If $a \in \sum F^{2}$ or if $\varphi$ is torsion, then $\langle 1,-a\rangle \otimes \varphi \in I_{t}^{n+1} F=0$, whence $a \varphi \cong \varphi$. Moreover, if $a \varphi \cong \varphi$ for every $a \in F^{\times}$, then obviously $\varphi$ is universal.

For a quadratic form $\varphi$ over $F$ and a field extension $K / F$ we denote by $\varphi_{K}$ the quadratic form with the same coefficients considered over $K$.

Let $K / F$ be a quadratic extension such that $K$ is nonreal. It is known that, for any $n \in \mathbb{N}$, one has $I^{n+1} K=0$ if and only if $I_{t}^{n+1} F=0$ and $s t(F) \leq n$ (cf. $[9,(2.6)]$ ).

\section{Signatures}

Let $\varphi$ be a quadratic form over $F$. Assume that $F$ is real and let $P$ be an ordering of $F$. We denote by $\operatorname{sign}_{P}(\varphi)$ the signature of $\varphi$ at $P$. We say that $\varphi$ is indefinite at $P$ if $\varphi$ represents nonzero elements of $P$ and of $-P$, or equivalently, if $\left|\operatorname{sign}_{P}(\varphi)\right|<\operatorname{dim}(\varphi)$. The form $\varphi$ over $F$ is totally indefinite if it is indefinite at every ordering of $F$. Every weakly isotropic form is totally indefinite, while the converse is not true in general. In fact, every totally indefinite form over $F$ is weakly isotropic if and only if $F$ is a $S A P$-field.

The form $\varphi$ over $F$ is said to be effectively diagonalizable if it has a diagonalization $\varphi=\left\langle a_{1}, \ldots, a_{n}\right\rangle$ where $a_{1} \ldots, a_{n} \in F^{\times}$are such that for every ordering $P \in X(F)$ and $1 \leq i \leq j \leq n$ one has $\operatorname{sign}_{P}\left(a_{i}\right) \geq \operatorname{sign}_{P}\left(a_{j}\right)$. It is easy to see that any effectively diagonalizable form is totally indefinite if and only if it contains a subform $\langle a,-b\rangle$ with $a, b \in \sum F^{2}$; in particular such a form is weakly isotropic. The field $F$ is said to have the $E D$-property or to be an $E D$-field if every quadratic form over $F$ is effectively diagonalizable. Since over an $E D$-field any totally indefinite form is weakly isotropic, it is clear that the $E D$-property implies $S A P$. The field $\mathbb{Q}((t))$ is a $S A P$-field but not an $E D$-field. Hence the $E D$-property is even more restrictive than $S A P$. Examples of $E D$-fields are number fields and extensions of transcendence degree one of a real closed field. Furthermore, any nonreal field 
as well as any algebraic extension of a uniquely ordered field has the $E D$-property. $E D$-fields were introduced by Prestel and Ware [22]. There it was also shown that, if $F$ is an $E D$-field, then so is every quadratic extension of $F$.

Lemma 2.2 Assume that $F$ is a real field of stability index $s t(F)<\infty$. For any quadratic form $\varphi$ over $F$ and any $r \geq s t(F)$, there exists a quadratic form $\varphi^{\prime}$ over $F$ such that $\varphi \equiv \varphi^{\prime}$ $\bmod I^{r} F$ and $0 \leq \operatorname{sign}_{P}\left(\varphi^{\prime}\right)<2^{r}$ for all $P \in X(F)$; moreover, the form $\varphi^{\prime}$ is unique up to $I_{t}^{r} F$.

Proof Since we may add to $\varphi$ any multiple of the form $2^{r} \times\langle 1\rangle$ without changing its class modulo $I^{r} F$, we may assume that $\operatorname{sign}_{P}(\varphi) \geq 0$ for all $P \in X(F)$. If now $\operatorname{sign}_{P}(\varphi)<2^{r}$ for all $P \in X(F)$, then we may take $\varphi^{\prime}=\varphi$. Let $H$ denote the set of those orderings $P$ of $F$ where $\operatorname{sign}_{P}(\varphi) \geq 2^{r}$ and assume now that $H \neq \emptyset$. Note that $H$ is a clopen subset of $X(F)$. We write $H=H_{1} \cup \ldots \cup H_{m}$ with pairwise disjoint basic clopen sets $H_{1} \ldots, H_{m}$. Since $r \geq s t(F)$, for $1 \leq i \leq m$ there is an $r$-fold Pfister form $\pi_{i}$ over $F$ such that $H_{i}=\left\{P \in X(F) \mid \operatorname{sign}_{P}\left(\pi_{i}\right)=2^{r}\right\}$. We put $\psi=\pi_{1}+\cdots+\pi_{m}$. Then $\psi \in I^{r} F$ and

$$
\operatorname{sign}_{P}(\psi)=\left\{\begin{array}{cl}
2^{r} & \text { for } P \in H \\
0 & \text { for } P \in X(F) \backslash H .
\end{array}\right.
$$

It follows that $\varphi \perp-\psi \equiv \varphi \bmod I^{r} F$ and that $\varphi \perp-\psi$ has only nonnegative signatures, while the maximal signature of $\varphi \perp-\psi$ is strictly smaller than the maximal signature of $\varphi$. Continuing this signature reduction process one finds a form $\varphi^{\prime}$ with the desired property.

Assume finally that $\varphi^{\prime \prime}$ is a second form with the properties requested for $\varphi^{\prime}$. Then the form $\varphi^{\prime} \perp-\varphi^{\prime \prime}$ belongs to $I^{r} F$ and $\left|\operatorname{sign}_{P}\left(\varphi^{\prime} \perp-\varphi^{\prime \prime}\right)\right|<2^{r}$ for every $P \in X(F)$. This implies that $\operatorname{sign}_{P}\left(\varphi^{\prime} \perp-\varphi^{\prime \prime}\right)=0$ for all $P \in X(F)$. Therefore $\varphi^{\prime} \perp-\varphi^{\prime \prime}$ is a torsion form in $I^{r} F$.

Assume that $F$ is a real field. Given a preordering $T$ of $F$, we denote

$$
I_{T} F=\left\{\varphi \in W F \mid \operatorname{sign}_{P}(\varphi)=0 \text { for all } P \in X_{T}(F)\right\} .
$$

It is well-known that $I_{T} F$ is equal to the ideal in $W F$ which is generated by the forms $\langle 1,-a\rangle$ with $a \in T^{\times}$. For $T=\sum F^{2} \cup\{0\}$ one has $I_{T} F=I_{t} F$, by Pfister's Local-Global Principle. Using (2.2) we retrieve a result in [13].

Corollary 2.3 Let $F$ be a real field, $T$ a preordering of $F$, and $r \geq \operatorname{st}(F)$. Then a quadratic form $\varphi$ over $F$ belongs to the ideal $I^{r} F+I_{T} F$ if and only if $\operatorname{sign}_{P}(\varphi) \equiv 0 \bmod 2^{r}$ holds for every $P \in X_{T}(F)$.

Proof The condition on the signatures is necessary, by the definition of $I_{T} F$ and since $I^{r} F$ is generated by $r$-fold Pfister forms, which can only have signature equal to 0 or $2^{r}$.

Assume now that $\varphi$ satisfies the condition on the signatures. By (2.2) there exists a form $\varphi^{\prime}$ over $F$ such that $\varphi \equiv \varphi^{\prime} \bmod I^{r} F$ and $\left|\operatorname{sign}_{P}\left(\varphi^{\prime}\right)\right|<2^{r}$ for any $P \in X(F)$. Since we have $\varphi^{\prime} \perp-\varphi \in I^{r} F$, the necessity of the condition shown above yields that $\operatorname{sign}_{p}\left(\varphi^{\prime} \perp-\varphi\right)$ is divisible by $2^{r}$ for any $P \in X_{T}(F)$, and then the same holds for $\operatorname{sign}_{p}\left(\varphi^{\prime}\right)$. This means that $\operatorname{sign}_{P}\left(\varphi^{\prime}\right)=0$ for every $P \in X_{T}(F)$. Thus $\varphi^{\prime} \in I_{T} F$ and $\varphi \in I^{r} F+I_{T} F$.

Remark 2.4 The equivalence formulated in (2.3) is actually valid for any $r \in \mathbb{N}$. It was conjectured by Marshall and is also known as Lam's Open Problem B. For $r \leq 2$ the equivalence is easy to show. For $2<r<s t(F)$, however, the proof uses a deep result from [19]. A short explanation of how to derive the equivalence from that result can be found in [16]. 


\section{The Clifford invariant}

The link between quadratic forms and central simple algebras of exponent 2 is established by the Clifford invariant. This connection has been fruitfully exploited to the study of quadratic forms, in particular by Merkurjev in his construction of a nonreal field of cohomological 2-dimension 2 and $u$-invariant $2 k$, for arbitrary $k \geq 2$.

\section{Merkurjev's Theorem}

For the definition and the basic properties of the Clifford invariant, especially about the interpretation in terms of the Clifford algebra, we refer to [14, Chap. V]. Let us briefly recall what we shall need.

Recall that $\operatorname{char}(F) \neq 2$. The Clifford invariant is a map

$$
c: W F \longrightarrow \mathrm{Br}_{2}(F) \text {. }
$$

If $\varphi$ is of even dimension then $c(\varphi)$ is the class of the Clifford algebra $C(\varphi)$ of $\varphi$. In general, the Clifford invariant $c(\varphi)$ may be calculated explicitly from any diagonalization of $\varphi$ by the formulas in [24, Chap. 2, Sect. 12]. Most important for us, for $a, b \in F^{\times}$the twofold Pfister form $\langle\langle a, b\rangle\rangle=\langle 1,-a,-b, a b\rangle$ corresponds via this map to the quaternion algebra $(a, b)_{F}$. While $c$ is not itself a homomorphism, simple calculations show that its restriction to $I^{2} F$ is a homomorphism and that it is trivial on $I^{3} F$.

Merkurjev [17] proved the following theorem.

Theorem 3.1 (Merkurjev) The homomorphism $c: I^{2} F \longrightarrow \mathrm{Br}_{2}(F)$ is surjective and its kernel is equal to $I^{3} \mathrm{~F}$.

The surjectivity can be reformulated by saying that every central simple algebra of exponent 2 is Brauer equivalent to a tensor product of quaternion algebras. In particular, if $F$ is quadratically closed, then $\mathrm{Br}_{2}(F)$ is trivial.

An algebraic extension $K / F$ is called a 2-extension if it can be embedded into a quadratic closure of $F$. For any division algebra $D$ of exponent 2 over $F$, there is a finite 2-extension $K / F$ such that $D_{K}$ is split. In fact (3.1) implies that $K / F$ can be chosen as a multiquadratic extension, i.e. a Galois extension with Galois group $(\mathbb{Z} / 2 \mathbb{Z})^{r}$ for some $r \in \mathbb{N}$.

Question 3.2 Let $D$ be a division algebra of exponent 2 over $F$. Is there a maximal subfield $K$ of $D$ such that $K / F$ is a 2-extension?

Remark 3.3 If $D$ is of index $2^{m}$ with $m \leq 3$, then there is a maximal subfield $K$ of $D$ such that $K / F$ is a Galois extension with group $(\mathbb{Z} / 2 \mathbb{Z})^{m}$, so in particular a 2 -extension. This is trivial for $m=1$ and follows from Albert's theorem about biquaternion algebras when $m=2$. For $m=3$ this is a theorem due to Rowen [23, p. 279, Exercise 32].

Nonreal central simple algebras

Over real fields there are variants of Merkurjev's Theorem in relation to preorderings. The statement below was derived in [15, Theorem 4, Corollary] from a conjecture that is settled in the meantime. For the reader's convenience we give the argument from a current point of view.

Theorem 3.4 (Marshall) Let $T$ be a preordering of $F$. The kernel of the map $\mathrm{Br}_{2}(F) \longrightarrow$ $\prod_{P \in X_{T}(F)} \mathrm{Br}_{2}\left(F_{P}\right)$ is generated by the classes of quaternion algebras of the form $(t, u)_{F}$ with $t \in T^{\times}$and $u \in F^{\times}$. 
Proof Any quaternion algebra $(t, u)_{F}$ with $t \in T$ and $u \in F^{\times}$becomes split over $F_{P}$ for any ordering $P \in X_{T}(F)$, because $T \subset F_{P}{ }^{2}$. Hence, such a quaternion algebra represents an element of the kernel of the map in the statement. Assume now that $\alpha$ is an element in this kernel. By Merkurjev's Theorem, there exists a form $\varphi \in I^{2} F$ such that $\alpha=c(\varphi)$. Since $\alpha$ lies in the kernel of the map in the statement, for any $P \in X_{T}(F)$ we have $c\left(\varphi_{F_{P}}\right)=0$, so $\varphi_{F_{P}} \in I^{3} F_{P}$ and therefore $\operatorname{sign}_{P}(\varphi) \equiv 0 \bmod 8$. By (2.4) we thus have $\varphi \equiv \psi \bmod I^{3} F$ for some form $\psi$ in $I^{2} F$ such that $\operatorname{sign}_{P}(\psi)=0$ for all $P \in X_{T}(F)$. Then $c(\psi)=c(\varphi)=\alpha$. On the other hand, $\psi$ is Witt equivalent to $\perp_{i=1}^{r}\left\langle\left\langle t_{i}, u_{i}\right\rangle\right\rangle$ for certain $t_{1}, \ldots, t_{r} \in T^{\times}$and $u_{1}, \ldots, u_{r} \in F^{\times}$. Hence, the class $\alpha=c(\psi)$ is represented by $\bigotimes_{i=1}^{r}\left(t_{i}, u_{i}\right)_{F}$.

We say that a central simple algebra $A$ over $F$ is nonreal, if its class lies in the kernel of

$$
\operatorname{Br}(F) \rightarrow \prod_{P \in X(F)} \operatorname{Br}\left(F_{P}\right)
$$

that is, if $A$ splits over every real closure of $F$. If $F$ is nonreal then every central simple algebra over $F$ is nonreal. Note that, for any central simple algebra $A$ over $F$, all even powers $A^{\otimes 2 m}(m \geq 0)$ are nonreal; this is immediate from the fact that the Brauer group of a real closed field is $\mathbb{Z} / 2 \mathbb{Z}$. Let us reformulate (3.4) for the canonical preordering $T=\sum F^{2} \cup\{0\}$.

Corollary 3.5 A central simple algebra of exponent at most 2 over $F$ is nonreal if and only if it is Brauer equivalent to a product of quaternion algebras of the form $(s, u)_{F}$ with $s \in \sum F^{2}$ and $u \in F^{\times}$.

Remark 3.6 The proof of (3.4) made use of the solution to Lam's Open Problem B (2.4). It might be seen as a challenge to obtain an elementary proof of (3.5). For the case where $s t(F) \leq 3$, a complete argument is given by $(2.3)$.

Corollary 3.7 A central simple algebra over $F$ of exponent $2^{r}(r \geq 0)$ is nonreal if and only if it splits over the pythagorean closure of $F$.

Proof Since the pythagorean closure of $F$ is contained in every real closure of $F$, the condition is obviously sufficient. To prove that it is necessary we may assume that $F$ is pythagorean. Now we have to show that every nonreal algebra $A$ over $F$ of exponent a power of 2 is split. By (3.5) $A$ cannot have exponent 2. But if $\exp (A)=2^{r}$ with $r>0$ then $A^{\otimes 2^{r-1}}$ is nonreal and of exponent 2 , which is impossible. Therefore $\exp (A)=1$, i.e. $A$ is split.

Remark 3.8 Assume that $I_{t}^{2} F=0$ (e.g. $F$ pythagorean). Then for any $s \in \sum F^{2}$ and $t \in F^{\times}$ the quaternion algebra $(s, t)_{F}$ is split. It follows then from (3.5) that there is no nonreal central simple algebra of exponent 2 over $F$. This implies that there is no central simple algebra of exponent divisible by 4 . The latter statement is actually equivalent to $I_{t}^{2} F=0$, by $[4,(3.3)]$.

Lemma 3.9 Assume that $I_{t}^{3} F=0$. Then every nonreal quaternion algebra over $F$ is of the form $(s, u)_{F}$ with $s \in \Delta_{F}(\langle 1,1\rangle)$ and $u \in F^{\times}$.

Proof Let $Q$ be a quaternion algebra over $F$ and let $\pi$ be its norm form. Assuming that $Q$ is nonreal, we have that $\pi$ is a torsion form. Since $l_{t}^{3} F=0$, it follows that $\pi$ contains a two-dimensional subform $\beta$ such that $2 \times \beta$ is hyperbolic (cf. [3, (3.5)]). Then $s=d_{ \pm}(\beta) \in$ $\Delta_{F}(\langle 1,1\rangle)$ and there is $u \in F^{\times}$such that $\pi \cong\langle\langle s, u\rangle\rangle$, whence $Q \cong(s, u)_{F}$.

Without any hypothesis on $F$, the conclusion of the lemma would be wrong. To emphasize this, we recall a famous example from [2]. 
Example 3.10 (Arason-Pfister) We consider $Q=\left(X, 1+Y^{2}-3 X\right)_{F}$ over the field $F=$ $\mathbb{Q}(X, Y)$. This quaternion algebra is not isomorphic to $(s, u)_{F}$ for any $s \in \sum F^{2}$ and $u \in F^{\times}$ (cf. [2]). However, $Q$ is Brauer equivalent to $\left(3,1+Y^{2}-3 X\right)_{F} \otimes_{F}\left(1+Y^{2}, 3 X\left(1+Y^{2}-3 X\right)\right)_{F}$ and therefore splits over every real closure of $F$.

\section{Associated forms}

So far we collected results on central simple algebras describing them up to Brauer equivalence. However, one aim of this article is to understand their intrinsic structure. The results that will be obtained depend on hypotheses on the base field $F$ by conditions on $I^{3} F$.

Let $A$ be a central simple algebra over $F$. We say that a quadratic form $\varphi$ and a central simple $F$-algebra $A$ are associated with each other if $\varphi \in I^{2} F$ and if the Clifford algebra $C(\varphi)$ is Brauer equivalent to $A$. By Merkurjev's Theorem, every central simple algebra $A$ over $F$ of exponent 1 or 2 is associated with some quadratic form in $I^{2} F$.

Proposition 3.11 A central simple algebra A of exponent 1 or 2 over $F$ is nonreal if and only if $A$ is associated with some form in $I_{t}^{2} F$.

Proof If $A$ is associated with a form $\varphi \in I_{t}^{2} F$, then clearly $A$ is nonreal. The converse implication follows from (3.5), since for any $s \in \sum F^{2}$ and any $u \in F^{\times}$the quaternion algebra $(s, u)_{F}$ is associated with $\langle\langle s, u\rangle\rangle \in I_{t}^{2} F$ and since $c: I^{2} F \longrightarrow \operatorname{Br}_{2}(F)$ is a homomorphism.

The following statements are well-known. Statement (b) has been a vital ingredient of Merkurjev's $u$-invariant construction.

Lemma 3.12 Let $\varphi \in I^{2} F$ with $\operatorname{dim}(\varphi) \geq 4$. Let $m \geq 1$ be such that $\operatorname{dim}(\varphi)=2 m+2$ and let $A$ be the central simple $F$-algebra of degree $2^{m}$ such that $C(\varphi) \cong M_{2}(A)$. Then the following hold:

(a) A is decomposable into a product of $m$ quaternion algebras.

(b) If $A$ is a division algebra, then $\varphi$ is anisotropic.

(c) If $\varphi \cong\langle a, b, c\rangle \perp \psi$ with $a, b, c \in F^{\times}$and a form $\psi$ of dimension $2 m-1$ over $F$, then $A \cong(-a b,-a c) \otimes B$ where $B$ is the central simple $F$-algebra such that $M_{2}(B) \cong C(\psi \perp\langle-a b c\rangle)$. Furthermore, the form $\psi \perp\langle-a b c\rangle \in I^{2} F$ is associated with $B$.

Proof Part (c) follows from the results in [14, Chap. V, Sect. 3], and it immediately yields (a) and (b).

Corollary 3.13 Let $D$ be a division algebra of exponent 2 over $F$ and let $m \geq 1$ such that $\operatorname{ind}(D)=2^{m}$. Then any form $\varphi \in I^{2} F$ associated with $D$ satisfies $\operatorname{diman}(\varphi) \geq 2 m+2$. Furthermore, $D$ is decomposable into a product of quaternion algebras if and only if there exists a form $\varphi \in I^{2} F$ associated with $D$ such that $\operatorname{dim}(\varphi)=2 m+2$.

Proof This is immediate from (3.12).

Lemma 3.14 Assume that st $(F) \leq 3$. Then any central simple algebra A of exponent 1 or 2 over $F$ is associated with a form $\varphi \in I^{2} F$ such that $\operatorname{sign}_{P}(\varphi) \in\{0,4\}$ for any $P \in X(F)$. Moreover, $\varphi$ is determined by $A$ up to $I_{t}^{3} F$, and for any $P \in X(F)$ one has

$$
\operatorname{sign}_{P}(\varphi)= \begin{cases}0 & \text { if } A_{F_{P}} \text { is split } \\ 4 & \text { otherwise. }\end{cases}
$$


Proof Let $\varphi \in I^{2} F$ be a form associated with $A$. Then $\varphi \equiv \varphi^{\prime} \bmod I^{3} F$ for some form $\varphi^{\prime} \in I^{2} F$ with $0 \leq \operatorname{sign}_{P}\left(\varphi^{\prime}\right)<8$ for all $P \in X(F)$. We now can replace $\varphi$ by $\varphi^{\prime}$ and conclude that all the signatures of this form are 0 or 4 . The second part of the statement is obvious.

\section{Witt index and quadratic extensions}

Some statements on quadratic forms can be proven by induction on the dimension of a form by applying the induction hypothesis to the anisotropic part over a quadratic extension of the ground field. To apply this method we need some control on the Witt index of a form over a quadratic extension. In this section a couple of statements serving this purpose are collected. The results in this section depend vitally on the condition that $I_{t}^{3} F=0$.

Lemma 4.1 Let $\varphi$ be an anisotropic form over $F$ and $K=F(\sqrt{d})$ where $d \in F^{\times} \backslash F^{\times 2}$. Then

(a) $\varphi_{K}$ is isotropic if and only if $\varphi$ has a subform $\langle a,-a d\rangle$ for some $a \in F^{\times}$.

(b) $i\left(\varphi_{K}\right) \geq 2$ if and only if $\varphi$ has a subform similar to a twofold Pfister form which is split over $K$.

Proof See [24, (2.5.1)] for (a). Part (b) follows from (a).

A quadratic extension $K / F$ is said to be totally positive if $K=F(\sqrt{d})$ for some $d \in \sum F^{2}$.

Proposition 4.2 Assume that $I_{t}^{3} F=0$ and let $K / F$ be a totally positive quadratic extension. Let $\varphi$ be an anisotropic form over $F$, not equal to a twofold Pfister form. Then $i\left(\varphi_{K}\right) \leq 1$.

Proof Suppose that $i\left(\varphi_{K}\right) \geq 2$. By $(4.1, \mathrm{~b}) \varphi$ contains a form $\pi$ over $F$ which is similar to a twofold Pfister form and such that $\pi_{K}$ is split. Then $\pi \in I^{2} F$ and, as $K / F$ is totally positive, $\pi$ is torsion. Using $(2.1$, b) it follows that $\pi$ is universal and itself a twofold Pfister form. Since $\varphi$ is anisotropic we obtain that $\varphi \cong \pi$. This contradicts the hypothesis on $\varphi$.

The following statement is partially contained in $[9,(2.3)]$.

Proposition 4.3 Assume that $I_{t}^{3} F=0$. Let $K / F$ be a quadratic extension and $\varphi$ an anisotropic torsion form over $F$, not equal to a twofold Pfister form. Then $i\left(\varphi_{K}\right) \leq 1$.

Proof Suppose that $i\left(\varphi_{K}\right) \geq 2$. Then $\varphi \cong \psi \perp \pi$ for two forms $\psi$ and $\pi$ over $F$ where $\pi$ is similar to a twofold Pfister form such that $\pi_{K}$ is split. By the same arguments as in the last proof, $\pi$ is not torsion. Using that $I_{t}^{3} F=0$ and [3, (3.5)], we decompose $\psi \cong \beta \perp \gamma$ with a torsion form $\beta$ and a strongly anisotropic form $\gamma$ over $F$. Since $\pi$ and $\gamma$ are strongly anisotropic while $\pi \perp \gamma$ is torsion, we have $\operatorname{dim}(\gamma)=\operatorname{dim}(\pi)=4$. We pick an element $a \in \Delta_{F}(\gamma)$. Then $\pi \perp\langle a\rangle$ is a five-dimensional subform of the eight-dimensional torsion form $\pi \perp \gamma$. A dimension argument shows that $\pi \perp\langle a\rangle$ is weakly isotropic. But $\pi \perp\langle a\rangle$ is similar to a subform of a threefold Pfister form $\rho$. As $\pi \perp\langle a\rangle$ is weakly isotropic, we obtain that $\rho \in I_{t}^{3} F=0$. Now a dimension argument yields that $\pi \perp\langle a\rangle$ is isotropic. This is in contradiction to $\pi \perp\langle a\rangle$ being a subform of the anisotropic form $\varphi$.

There is an application of (4.3) to the study of the $u$-invariant. In [6] the $u$-invariant of the field $F$ is defined as

$$
u(F)=\sup \{\operatorname{dim}(\varphi) \mid \varphi \text { anisotropic torsion form over } F\}
$$


Corollary 4.4 Assume that $I_{t}^{3} F=0$. Then for any quadratic extension $K / F$ one has $u(K) \geq$ $u(F)-2$.

Proof We may assume that $u(F)>4$. Let $\varphi$ be an arbitrary anisotropic torsion form over $F$ with $\operatorname{dim}(\varphi)>4$. Since $i\left(\varphi_{K}\right) \leq 1$ by (4.3), we obtain that $u(K) \geq \operatorname{diman}\left(\varphi_{K}\right) \geq$ $\operatorname{dim}(\varphi)-2$.

Remark 4.5 In [9], it was shown that if $I^{3} K=0$ for $K=F(\sqrt{-1})$, then $u(F) \leq 4 \lambda(K)+2$, where $\lambda(K)$ is the symbol length of $K$. Note that the condition that $I^{3} K=0$ implies on the one hand that $l_{i}^{3} F=0$, on the other hand that $u(K)=2 \lambda(K)+2$ (except in the case where $u(K)=1)$. Hence $u(F) \leq u(K)+2$, by $(4.4)$, and thus $u(F) \leq 2 \lambda(K)+4$.

Over $E D$-fields the conclusion in the last proposition is valid not only for torsion forms but more generally for totally indefinite forms. While in some disguise, the following statement is contained in $[9,(2.3)]$.

Proposition 4.6 (Hoffmann) Assume that $F$ has the ED-property and $I_{t}^{3} F=0$. Let $\varphi$ be an anisotropic, totally indefinite form over $F$, not equal to a twofold Pfister form. Then $i\left(\varphi_{K}\right) \leq 1$ for any quadratic extension $K / F$.

Proof Suppose that $K / F$ is a quadratic extension with $i\left(\varphi_{K}\right) \geq 2$. Then $\varphi$ contains a form $\pi$ similar to a twofold Pfister form. Let $\vartheta$ be the complement of $\pi$ in $\varphi$, i.e. $\varphi \cong \pi \perp \vartheta$. As seen before, $\pi$ is strongly anisotropic. Since $\varphi$ is totally indefinite it is weakly isotropic, because $F$ is an $E D$-field. Thus there exists an integer $m \geq 1$ such that $m \times \pi$ and $m \times(-\vartheta)$ represent a nonzero element $c$ in common. Since $-c$ is represented by $m \times \vartheta$, it follows from the $E D$-property of $F$ that $-t c \in \Delta_{F}(\vartheta)$ for some $t \in \sum F^{2}$. Then $\pi \perp\langle-t c\rangle$ is a subform of $\varphi$. From the choice of $c$ and $t$ it follows that $\pi \perp\langle-t c\rangle$ is weakly isotropic. But $\pi \perp\langle-t c\rangle$ is similar to a subform of a threefold Pfister form $\rho$. As $\pi \perp\langle-t c\rangle$ is weakly isotropic, we obtain that $\rho \in I_{t}^{3} F=0$. This yields that $\pi \perp\langle-t c\rangle$ is isotropic, contradicting that $\varphi$ is anisotropic.

The conclusion of the proposition would not hold anymore if instead of the $E D$-property we assumed only SAP.

Example 4.7 Let $k$ be a maximal extension of $\mathbb{Q}$ in $\mathbb{R}$ such that 2 is not a square in $k$. Then $k$ is uniquely ordered and has four square classes, given by \pm 1 and \pm 2 . Let $F=k((t))$, the formal power series field in one variable $t$ over $k$. Now $F$ has eight square classes, given by the set of representatives $\{ \pm 1, \pm 2, \pm t, \pm 2 t\}$. Moreover $F$ satisfies $S A P$ and $I_{t}^{3} F=0$. However $F$ is not an $E D$-field as the form $\langle t,-2 t\rangle$ cannot be effectively diagonalized.

Let $K=F(\sqrt{-1})$ and $m \geq 2$. The quadratic form $\varphi=\langle 1,-2\rangle \perp 2 m \times\langle t\rangle$ over $F$ is anisotropic, while $2 \times \varphi$ is isotropic. Hence $\varphi$ is totally indefinite and $i\left(\varphi_{K}\right)=m$.

Remark 4.8 Assume that $I_{i}^{3} F=0$. Let $K / F$ be a quadratic extension. One can ask whether $i\left(\varphi_{K}\right) \leq 1$ holds for every anisotropic form $\varphi$ over $F$ which is weakly isotropic and not a twofold Pfister form. The critical case is when the dimension is 6 . In fact, if $i\left(\varphi_{K}\right) \leq 1$ holds for every six-dimensional anisotropic weakly isotropic form $\varphi$ over $F$, then this bound holds actually for all anisotropic weakly isotropic forms except for twofold Pfister forms.

On the other hand, if there is a six-dimensional anisotropic weakly isotropic form $\varphi$ such that $i\left(\varphi_{K}\right) \geq 2$, then we can decompose $\varphi=\pi \perp \vartheta$ where $\pi$ is similar to a twofold Pfister form which splits over $K$ and $\vartheta$ is of dimension 2 . As seen before, $\pi$ is strongly anisotropic. Let $m \geq 1$. Since $I_{t}^{3} F=0$ it follows that $\pi$ and $m \times \pi$ represent exactly the same elements. In particular $\psi=\vartheta \perp m \times \pi$ is anisotropic, but weakly isotropic. Furthermore $i\left(\psi_{K}\right) \geq 2 m$, because $m \times \pi$ splits over $K$. 


\section{Canonical forms}

Given a quaternion algebra $Q$ over $F$ there is a canonical choice for a quadratic form $\varphi \in I^{2} F$ whose Clifford invariant gives the class of $Q$ in $\mathrm{Br}_{2}(F)$, the norm form of $Q$. In general, given a central simple algebra of exponent 2 over $F$, Merkurjev's Theorem only affirms the existence of a quadratic form whose Clifford invariant is the class of that algebra. Here we are interested in getting a good choice for this form. Several results in this section involve the two conditions that $l_{t}^{3} F=0$ and $s t(F) \leq 3$. Recall that, if $I^{3} F(\sqrt{-1})=0$, then $l_{t}^{3} F=0$ and $s t(F) \leq 2$, so the results apply in this case.

We call a nontrivial form $\varphi \in I^{2} F$ a canonical form if $\varphi$ is anisotropic and if $\operatorname{sign}_{P}(\varphi) \in$ $\{0,4\}$ for every $P \in X(F)$. This notion will be interesting essentially in the case where $I_{t}^{3} F=0$.

Proposition 5.1 Assume that $I_{t}^{3} F=0$. Given a central simple algebra A of exponent 2 over $F$, there is at most one canonical form associated with $A$.

Proof Assume that $\varphi_{1}$ and $\varphi_{2}$ are two forms over $F$ with these properties. Since $c\left(\varphi_{1}\right)=c\left(\varphi_{2}\right)$ in $\operatorname{Br}_{2}(F)$, it follows from (3.1) that $\varphi_{1} \perp-\varphi_{2} \in l^{3} F$. Since $\operatorname{sign}_{P}\left(\varphi_{1} \perp-\varphi_{2}\right) \in\{-4,0,4\}$ for all $P \in X(F)$, we conclude that $\varphi_{1} \perp-\varphi_{2} \in I_{t}^{3} F=0$. As $\varphi_{1}$ and $\varphi_{2}$ are both anisotropic, it follows that $\varphi_{1}=\varphi_{2}$.

Question 5.2 Is every division algebra of exponent 2 over $F$ associated with a canonical form?

Example 5.3 Let $Q$ be a nonsplit quaternion algebra over $F$. Then $Q$ is associated with its norm form, which is a canonical form over $F$.

Proposition 5.4 Let $D$ be a division algebra of exponent 2 over $F$. If $D$ is nonreal or, more generally, if there exists a quaternion algebra $Q$ over $F$ such that $D \otimes_{F} Q$ is nonreal, then $D$ is associated with a canonical form.

Proof Assume that $Q$ is a (possibly split) quaternion algebra over $F$ such that $D \otimes_{F} Q$ is nonreal. By (3.11), $D \otimes_{F} Q$ is associated with a form $\varphi \in I_{t}^{2} F$. Let $\pi$ denote the norm form of $Q$ and let $\psi$ be the anisotropic part of $\varphi \perp \pi$. Then $c(\psi)=c(\varphi)+c(\pi)$ is the class of $D$ in $\mathrm{Br}_{2}(F)$. It follows that $\psi$ is a canonical form and associated with $D$.

Proposition 5.5 If st $(F) \leq 3$, then any division algebra of exponent 2 over $F$ is associated with a canonical form. If in addition $I_{t}^{3} F=0$, then this gives a one-to-one correspondence between division algebras of exponent 2 and canonical forms over $F$.

Proof This follows from (3.14).

Remark 5.6 Let $\varphi$ be a canonical form over $F$. Then, for any field extension $K / F$ such that $\varphi_{K}$ is not hyperbolic, the anisotropic part $\left(\varphi_{K}\right)_{a n}$ is a canonical form over $K$. Moreover, if $\varphi$ is associated with the central simple algebra $A$ of exponent 2 over $F$ and if $I_{t}^{3} K=0$, then $\left(\varphi_{K}\right)_{a n}$ is the unique canonical form over $K$ associated with the algebra $A_{K}$.

Question 5.7 Assume that $I^{3} F(\sqrt{-1})=0$ and let $D$ be a biquaternion division algebra over $F$. Is the canonical form associated with $D$ always of dimension 6 , i.e. is it one of the Albert forms associated with $D$ ? 
Proposition 5.8 Let $D$ be a biquaternion division algebra over $F$. If $D$ contains a nonreal quadratic extension of $F$, then $D$ is associated to a canonical form which is an Albert form and represents 1 .

Proof Suppose $a \in F^{\times} \backslash F^{\times 2}$ is such that $F(\sqrt{a})$ is nonreal and embeds into $D$. Then $-a \in \sum F^{2}$ and there is an Albert form $\alpha$ associated with $D$ containing $\langle 1,-a\rangle$. It follows that $\alpha$ is a canonical form.

If $\varphi$ is a canonical form over $F$ which represents 1 , then we decompose $\varphi=\langle 1\rangle \perp \varphi^{\prime}$ and refer to $\varphi^{\prime}$ as the pure part of $\varphi$. Note that this is consistent with the notion of the pure part of a Pfister form.

Question 5.9 Assume that $I_{f}^{3} F=0$. Does every canonical form over $F$ represent 1 ?

We give some sufficient conditions.

Proposition 5.10 Assume that $I_{t}^{3} F=0$. Let $\varphi$ be a canonical form over $F$. If there exists a quadratic form $\psi$ over $F$ with $\operatorname{dim}(\psi)=4$ and such that $\operatorname{sign}_{P}(\varphi)=\operatorname{sign}_{P}(\psi)$ for every $P \in X(F)$, then $\varphi$ represents 1 .

Proof By $\left(2.1\right.$, a) it is sufficient to show that $\varphi$ represents an element of $\sum F^{2}$. As $I_{t}^{3} F=0$, any element of $\sum F^{2}$ is a sum of four squares in $F$. Therefore it suffices to show that $\varphi \perp-\langle 1,1,1,1\rangle$ is isotropic. We do this by induction on the dimension of $\varphi$.

Since $\varphi \in I^{2} F$, we have $\operatorname{dim}(\varphi) \geq 4$. Suppose that $\operatorname{dim}(\varphi)=4$. Then $\varphi \cong a \rho$ for some $a \in F^{\times}$and a twofold Pfister form $\rho$ over $F$. It is clear that $\operatorname{sign}_{P}(\varphi) \equiv \operatorname{sign} P(\rho) \bmod 8$ for any $P \in X(F)$. Since $\operatorname{sign}_{P}(\varphi)$ and $\operatorname{sign}_{P}(\rho)$ can each only be 0 or 4 for any $P \in X(F)$, we conclude that $\operatorname{sign}_{P}(\varphi)=\operatorname{sign}_{P}(\rho)$ for all $P \in X(F)$. Hence $\rho \perp-\varphi \cong\langle 1,-a\rangle \otimes \rho$ is a torsion threefold Pfister form, thus hyperbolic, which implies that $\varphi$ represents 1 .

Assume now that $\operatorname{dim}(\varphi)>4$. Let $d=d_{ \pm}(\psi)$. Since $\operatorname{dim}(\psi)=4$ and $\operatorname{sign}(\psi)=$ $\operatorname{sign}(\varphi) \in\{0,4\}$ for any $P \in X(F)$, it follows that $d \in \sum F^{2}$. Since we can multiply one coefficient of an arbitrary diagonalization of $\psi$ by $d$ without changing the properties required in the choice of $\psi$, we may actually assume that $d=1$, so that $\psi \in I^{2} F$. Then $\varphi \perp-\psi \in I_{t}^{2} F$ and $2 \times(\varphi \perp-\psi) \in I_{t}^{3} F=0$. By a dimension argument it follows that $2 \times \varphi$ is isotropic. Then $\varphi$ contains a two-dimensional torsion form $\beta$. Its discriminant $t=d_{ \pm}(\beta)$ is a sum of squares in $F$, but not itself a square, as $\varphi$ is anisotropic. We now shift the whole situation to the quadratic extension $K=F(\sqrt{t})$. Note that $\varphi_{K}$ is isotropic. Hence $i\left(\varphi_{K}\right)=1$ by (4.2). Let $\varphi^{\prime}$ be the anisotropic part of $\varphi_{K}$. As $i\left(\varphi_{K}\right)=1$ we have $\operatorname{dim}\left(\varphi^{\prime}\right)=\operatorname{dim}(\varphi)-2$. Note that $\operatorname{sign}_{P}\left(\varphi^{\prime}\right)=\operatorname{sign}_{P}\left(\psi_{K}\right)$ for any $P \in X(K)$. Moreover $I_{t}^{3} K=0$, because $I_{t}^{3} F=0$ and $K / F$ is a totally positive quadratic extension. We thus can apply the induction hypothesis and obtain that $\varphi^{\prime} \perp-\langle 1,1,1,1\rangle$ is isotropic over $K$. Therefore the Witt index of $\varphi \perp-\langle 1,1,1,1\rangle$ over $K$ is at least 2. By (4.2) this implies that $\varphi \perp-\langle 1,1,1,1\rangle$ is isotropic over $F$.

Corollary 5.11 Assume that $F$ is a SAP-field with $I_{t}^{3} F=0$. Then any canonical form over $F$ represents 1 .

Proof Since $F$ is a SAP-field, given a form $\varphi$ over $F$ with $\operatorname{sign}_{P}(\varphi) \in\{0,4\}$ for all $P \in X(F)$, we can choose $d \in F^{\times}$such that $\operatorname{sign}_{P}\left(\langle\langle-1, d\rangle)=\operatorname{sign}_{P}(\varphi)\right.$ for all $P \in X(F)$. Thus the statement follows from $(5.10)$.

Let $F_{\text {pyth }}$ denote the pythagorean closure of $F$. 
Corollary 5.12 Assume that $I_{t}^{3} F=0$ and $s t(F) \leq 3$. Let $D$ be a division algebra of exponent 2 over $F$. If $D$ is nonreal or, more generally, if ind $\left(D_{F_{\mathrm{pyth}}}\right) \leq 2$, then the canonical form associated with $D$ represents 1 .

Proof Let $\varphi$ be the canonical form associated with $D$. If $D_{F_{\mathrm{py}}}$ is split, then $\varphi$ is torsion, and the result follows from $(5.10)$ with $\psi=\left\langle\langle 1,1\rangle\right.$. Assume now that ind $\left(D_{F_{\mathrm{pyth}}}\right)=2$. Since $I_{t}^{3} F=0$, by [3, (3.5)] we have a decomposition $\varphi \cong \gamma \perp \vartheta$ where $\gamma$ is a strongly anisotropic form and $\vartheta$ is a torsion form over $F$. Then $\vartheta_{F_{\mathrm{pyth}}}$ is hyperbolic and $\gamma_{F_{\mathrm{pyth}}}$ is the unique canonical form associated with $D_{F_{\text {pyth }}}$. It follows that $\operatorname{dim}(\gamma)=4$ and $\operatorname{sign}_{P}(\varphi)=\operatorname{sign}_{P}(\gamma)$ for all $P \in X(F)$. By (5.10) applied with $\psi=\gamma$, this proves the claim.

\section{Decomposability}

We are going to show the equivalence of several properties for nonreal division algebras of exponent 2 over a field $F$ with $I^{3} F(\sqrt{-1})=0$. Under slightly stronger assumptions on $F$, we show in (6.4) that these properties are actually all satisfied. The key idea comes from $[11,(2.2)]$.

Proposition 6.1 Assume that $I^{3} F(\sqrt{-1})=0$. Let $D$ be a division algebra of exponent 2 over $F$. Let $m \geq 1$ be such that ind $(D)=2^{m}$. Assume either that $D$ is nonreal or that $F$ is an ED-field. Then the following are equivalent:

(1) $D$ is decomposable into a tensor product of quaternion algebras.

(2) D has a maximal subfield which is a 2-extension of $F$.

(3) The canonical form associated with $D$ has dimension $2 m+2$.

Proof The implications (3) $\Rightarrow(1) \Rightarrow(2)$ are obvious and hold without the last hypothesis above. It remains to show that (2) implies (3).

Assume that $D$ contains a maximal subfield $L$ such that $L / F$ is a 2-extension. Then $[L: F]=\operatorname{deg}(D)=2^{m}$. Let $\varphi$ be the canonical form associated with $D$. We will prove the equality $\operatorname{dim}(\varphi)=2 m+2$ by induction on $m$. Since ind $(C(\varphi))=\operatorname{ind}(D)=2^{m}$, we know that $\operatorname{dim}(\varphi) \geq 2 m+2$. If $m=1$ then $D$ is a quaternion algebra and $\varphi$ is its norm form, thus an anisotropic twofold Pfister form, so we have $\operatorname{dim}(\varphi)=4=2 m+2$. Let now $m>1$. Since $L / F$ is a 2-extension of degree $2^{m}$, there is a quadratic extension $K / F$ contained in $L / F$. Recall that $I^{3} F(\sqrt{-1})=0$ implies that $I^{3} K(\sqrt{-1})=0$. Moreover, if $F$ is an $E D$-field, then so is $K$. Let $C$ be the centralizer of $K$ in $D$. Then $C$ is a division algebra over $K$ of index $2^{m-1}$ and exponent 2 and Brauer equivalent to $D_{K}$. Therefore the canonical form associated with $C$ is the anisotropic part of $\varphi_{K}$. By induction hypothesis, since $C$ contains $L$ as a maximal subfield and $L / K$ is a 2-extension, we obtain that diman $\left(\varphi_{K}\right)=2(m-1)+2=2 m$. Note that either $\varphi$ is torsion or $F$ is an $E D$-field. Since $\varphi$ is anisotropic over $F$ and $\operatorname{dim}(\varphi)>4$ and since $I_{t}^{3} F=0$, we have $i\left(\varphi_{K}\right) \leq 1$ by (4.3) or (4.6), respectively. Therefore $\operatorname{dim}(\varphi)=$ $\operatorname{dim}\left(\varphi_{K}\right)=\operatorname{diman}\left(\varphi_{K}\right)+2 i\left(\varphi_{K}\right) \leq 2 m+2$. Since we know already that $\operatorname{dim}(\varphi) \geq 2 m+2$, equality follows.

Corollary 6.2 Assume that $I^{3} F(\sqrt{-1})=0$. Let $D$ be a nonreal division algebra of exponent 2 over $F$. If $D$ is isomorphic to a product of $m$ quaternion algebras, then there is even a decomposition

$$
D \cong\left(s_{1}, t_{1}\right)_{F} \otimes_{F} \cdots \otimes_{F}\left(s_{m}, t_{m}\right)_{F}
$$

where $s_{1}, \ldots, s_{m} \in \Delta_{F}(\langle 1,1\rangle)$ and $t_{1}, \ldots, t_{m} \in F^{\times}$. 
Proof Let $\varphi$ be the canonical form associated with $D$. By the hypothesis, $\operatorname{dim}(\varphi)=2 m+2$. As $D$ is nonreal, $\varphi$ is a torsion form. Thus $2 \times \varphi \in I_{i}^{3} F=0$. It follows that $\varphi$ contains a 2 dimensional form $\beta$ such that $2 \times \beta$ is hyperbolic. Hence, we can write $\varphi \cong\langle a,-a s,-a t\rangle \perp$ $\psi$ with a subform $\psi$ and some $s \in \Delta_{F}(\langle 1,1\rangle)$ and $a, t \in F^{\times}$. Using $(3.12, \mathrm{c})$ it follows that $D \cong(s, t)_{F} \otimes_{F} D^{\prime}$ where $D^{\prime}$ is a division algebra of exponent 1 or 2 and index $2^{m-1}$, associated with the form $\varphi^{\prime}=\langle-a s t\rangle \perp \psi$ of dimension $2 m$. If $m>1$, then $\varphi^{\prime}$ is anisotropic by $\left(3.12\right.$, b). Furthermore $\varphi^{\prime}$ is torsion. Hence $\varphi^{\prime}$ is the canonical form associated with $D^{\prime}$, and $D^{\prime}$ is nonreal and a product of $m-1$ quaternion algebras. We may now conclude by induction on $m$.

Corollary 6.3 Assume that $I^{3} F(\sqrt{-1})=0$ and let $D$ be a division algebra of exponent 2 and of index 8 over $F$. If $D$ is nonreal or if $F$ is an ED-field, then $D$ is isomorphic to a product of quaternion algebras.

Proof By (3.3), $D$ contains a maximal subfield which is a 2-extension of $F$. Hence the statement follows from (6.1).

A positive answer to Question (3.2) for fields $F$ with $I^{3} F(\sqrt{-1})=0$ would yield that the conditions (1)-(3) in (6.1) are not only equivalent but actually hold altogether.

Given a field $K$ of characteristic different from 2 , let

$$
c d_{2}(K)=\sup \left\{m \in \mathbb{N} \mid I^{m} L \neq 0 \text { for some finite extension } L / K\right\} .
$$

In view of the Milnor Conjecture, proven by Voevodsky [25], this actually agrees with the usual definition of the cohomological 2-dimension of $K$, where instead of $I^{m} L \neq 0$ one would have to read $H^{m}\left(L, \mu_{2}\right) \neq 0$. However, the definition taken here is closer to the way we are going to use it.

Theorem 6.4 Assume that $c d_{2}(F(\sqrt{-1})) \leq 2$ and let $D$ be a central division algebra of exponent 2 over $F$. Assume either that $D$ is nonreal or that every algebraic extension of $F$ is an ED-field. Then D is decomposable into a product of quaternion algebras.

Proof Let $\varphi$ be the canonical form of $D$. Let $m \geq 1$ be the integer such that $\operatorname{ind}(D)=2^{m}$. In view of (6.1) it suffices to show that $\operatorname{dim}(\varphi)=2 m+2$.

We choose a maximal subfield $L$ of $D$. Note that $[L: F]=2^{m}$ and that $L / F$ is separable since $\operatorname{char}(F) \neq 2$. Using Galois theory we may choose a finite extension of odd degree $M / F$ (automatically linearly disjoint to $L / F$ ) such that $L M / M$ is a 2-extension (where $L M$ denotes the composite). Note that $D_{M}$ is still a division algebra, hence of index $2^{m}$, because the degree $[M: F]$ is prime to ind $(D)$. The canonical form associated with $D_{M}$ is $\varphi_{M}$, because this form is anisotropic, by Springer's Theorem. Since $L M$ is a maximal subfield of the algebra $D_{M}$ and a 2 -extension of its center $M,(6.1)$ shows that $\operatorname{dim}(\varphi)=\operatorname{dim}\left(\varphi_{M}\right)=2 m+2$.

Corollary 6.5 Assume that $c d_{2}(F(\sqrt{-1})) \leq 2$. Let $D$ be a nonreal division algebra of exponent 2 over $F$ and $\varphi=\langle 1\rangle \perp \varphi^{\prime}$ the canonical form associated with $D$.

(a) For any $a \in F^{\times} \backslash-F^{\times 2}$, the algebra $D_{F(\sqrt{-a})}$ has nontrivial zero-divisors if and only if $\varphi_{F(\sqrt{-a})}$ is isotropic, if and only if $a \in \Delta_{F}\left(\varphi^{\prime}\right)$.

(b) Let $E$ be another nonreal division algebra of exponent 2 over $F$ with associated canonical form $\psi=\langle 1\rangle \perp \psi^{\prime}$. Then $D \otimes_{F} E$ is a division algebra if and only if $\Delta_{F}\left(\varphi^{\prime}\right) \cap$ $\Delta_{F}\left(\psi^{\prime}\right)=\emptyset$. 
Proof The first equivalence in (a) follows from (5.6), (6.1), and (6.4). Moreover, if $a \in$ $\Delta_{F}\left(\varphi^{\prime}\right)$ then certainly $\varphi_{F(\sqrt{-a})}$ is isotropic. Conversely, if $\varphi_{F(\sqrt{-a})}$ is isotropic, then $\varphi$ contains a subform similar to $\langle 1, a\rangle$, and using $(2.1, b)$ and Witt cancellation it follows that $\varphi^{\prime}$ represents $a$. This shows the second equivalence stated in (a).

To prove (b), we first observe that $D \otimes_{F} E$ is associated with $\varphi^{\prime} \perp-\psi^{\prime}$ and that this is a torsion form. We may assume that $D \otimes_{F} E$ is not split, so that $\varphi^{\prime} \perp-\psi^{\prime}$ is not hyperbolic. Since $D$ and $E$ are both nonreal, the canonical form associated with the $F$-algebra $D \otimes_{F} E$ is the anisotropic part of $\varphi^{\prime} \perp-\psi s^{\prime}$. Let $m, n \in \mathbb{N}$ be such that ind $(D)=2^{m}$ and ind $(E)=2^{n}$. Then $\operatorname{dim}(\varphi)=2 m+2$ and $\operatorname{dim}(\psi)=2 n+2$, thus $\operatorname{dim}\left(\varphi^{\prime} \perp-\psi^{\prime}\right)=2(m+n)+2$. Using (6.1), it follows that ind $\left(D \otimes_{F} E\right)=2^{m+n}$ if and only if $\varphi^{\prime} \perp-\psi^{\prime}$ is anisotropic, that is, if and only if $\varphi^{\prime}$ and $\psi^{\prime}$ do not represent any nonzero element in common.

Conjecture 6.6 If $I_{i}^{3} F=0$, then every division algebra of exponent 2 over $F$ is decomposable into a tensor product of quaternion algebras.

\section{Lengths and heights}

We are going to study decomposition lengths of division algebras of exponent 2 and compare them to heights of associated quadratic forms.

For a central simple $F$-algebra $A$ of exponent 1 or 2 we denote by $t(A)$ the least number $t \geq 0$ such that $A$ is Brauer equivalent to a product of $t$ quaternion algebras; by (3.1) such a number $t$ always exists. Note that $t(A)=0$ if and only if $\exp (A)=1$, that is, if $A$ is split. The number $t(A)$ is called the decomposition length of $A$.

In view of (3.4) we define a second invariant for nonreal central simple algebras. Given a nonreal central simple algebra of exponent 1 or 2 over $F$, we denote by $t^{\prime}(A)$ the least number $t \geq 0$ such that $A$ is Brauer equivalent to a tensor product of $t$ quaternion algebras of the type $(s, x)_{F}$ with $s \in \sum F^{2}$ and $x \in F^{\times}$. We may call $t^{\prime}(A)$ the nonreal decomposition length of $A$. Obviously $t^{\prime}(A) \geq t(A)$. As (3.10) showed, it may happen that $t^{\prime}(A)>t(A)$.

Conjecture 7.1 If $I_{t}^{3} F=0$, then for any nonreal central simple algebra $A$ of exponent 2 over $F$ one has $t^{\prime}(A)=t(A)$.

By (6.2) this is true under the stronger hypothesis that $I^{3} F(\sqrt{-1})=0$. Using a variation of the arguments in $[9,(2.14)]$ we can verify the conjecture for small values.

Proposition 7.2 Assume that $I_{t}^{3} F=0$. For any nonreal central simple algebra A of exponent 2 over $F$ with $t(A) \leq 4$ one has $t(A)=t^{\prime}(A)$

Proof On the one hand, there is an anisotropic form $\varphi \in I^{2} F$ of dimension $2 t(A)+2$ associated with $A$. On the other hand, the canonical form $\varphi^{\prime}$ associated with $A$ has dimension $2 t^{\prime}(A)+2$. Since we know that $t^{\prime}(A) \geq t(A)$, in order to prove the statement, we just need to show that $\operatorname{dim}(\varphi) \geq \operatorname{dim}\left(\varphi^{\prime}\right)$.

As $c(\varphi)=[A]=\bar{c}\left(\varphi^{\prime}\right),(3.1)$ yields that $\varphi \perp-\varphi^{\prime} \in I^{3} F$. We denote by $\pi$ the anisotropic part of $\varphi \perp-\varphi^{\prime}$. If $\pi$ is trivial, then $\varphi \cong \varphi^{\prime}$ and we are done. Assume that $\pi$ is nontrivial. Then $\operatorname{dim}(\pi) \geq 8$ by the Arason-Pfister Hauptsatz. On the other hand, $I_{i}^{3} F=0$ yields that $2 \times \varphi^{\prime}$ is hyperbolic, thus $2 \times \varphi$ is Witt equivalent to $2 \times \pi$ and in particular diman $(2 \times \pi) \leq$ $2(2 t(A)+2) \leq 20$. Since $2 \times \pi \in I^{4} F$, it follows now from [8] that the anisotropic part of $2 \times \pi$ is similar to some fourfold Pfister form $\rho$ over $F$. Moreover, $\rho$ then is of the form $2 \times \pi^{\prime}$ for some threefold Pfister form $\pi^{\prime}$ over $F$. Since $I_{f}^{3} F=0$, we obtain $\pi^{\prime} \cong \pi$, whence $\pi$ is a threefold Pfister form. 
Now $\varphi \perp-\pi$ is Witt equivalent to $\varphi^{\prime}$. Therefore $\operatorname{dim}(\varphi)<\operatorname{dim}\left(\varphi^{\prime}\right)$ would imply that $\varphi^{\prime}$ contains a five-dimensional Pfister neighbour of $\pi$. This is impossible by (4.3), as $\varphi^{\prime}$ is a torsion form.

For a quadratic form $\varphi$ over $F$ we define the 'correction term'

$$
\varepsilon(\varphi)= \begin{cases}2 & \text { if } \operatorname{dim}(\varphi) \text { is even and } d_{ \pm}(\varphi)=1 \\ 0 & \text { if } \operatorname{dim}(\varphi) \text { is even and } d_{ \pm}(\varphi) \neq 1 \\ 1 & \text { if } \operatorname{dim}(\varphi) \text { is odd }\end{cases}
$$

Note that $\varepsilon(\varphi)$ depends only on the Witt equivalence class of $\varphi$. We further put $t(\varphi)=t(c(\varphi))$, and similarly $t^{\prime}(\varphi)=t^{\prime}(c(\varphi))$ in case $\varphi$ is torsion.

Lemma 7.3 If $\varphi$ is not hyperbolic, then $t(\varphi) \leq \frac{1}{2} \cdot(\operatorname{diman}(\varphi)-\varepsilon(\varphi))$.

Proof If $\operatorname{dim}(\varphi) \leq 3$, then $t(\varphi) \leq 1$ and the inequality is easily checked. We assume now that $\operatorname{dim}(\varphi)>3$ and write $\varphi \cong \psi \perp\langle a, b, c\rangle$ for some form $\psi$ over $F$ and $a, b, c \in F^{\times}$. We can further assume that $\varphi$ is anisotropic. Let $\varphi^{\prime}=\psi \perp\langle-a b c\rangle$. Note that diman $\left(\varphi^{\prime}\right) \leq$ $\operatorname{diman}(\varphi)-2, \varepsilon\left(\varphi^{\prime}\right)=\varepsilon(\varphi)$, and $c(\varphi)=c\left(\varphi^{\prime}\right)+\left[(-a b,-a c)_{F}\right]$ in $\operatorname{Br}_{2}(F)$, whence $t(\varphi) \leq t\left(\varphi^{\prime}\right)+1$. The statement now follows by applying the induction hypothesis to $\varphi^{\prime}$.

In [3] a field $F$ was called a WD-field if every quadratic form $\varphi$ over $F$ can be decomposed into $\varphi \cong \varphi_{s} \perp \varphi_{t}$ where $\varphi_{s}$ is a strongly anisotropic form and $\varphi_{i}$ a torsion form over $F$. Obviously, any nonreal field is a $W D$-field. If $F$ is real, then $F$ is a $W D$-field if and only if every weakly isotropic form over $F$ contains a two-dimensional torsion form. Any ficld $F$ with $I_{f}^{3} F=0$ is a $W D$-field, by $[3,(3.5)]$.

Lemma 7.4 Assume that $F$ is a WD-field. For any non-hyperbolic torsion form $\varphi$ over $F$ one has $t(\varphi) \leq t^{\prime}(\varphi) \leq \frac{1}{2} \cdot(\operatorname{diman}(\varphi)-\varepsilon(\varphi))$.

Proof We only need to show the second inequality. The proof goes along the same lines as in (7.3), now using in addition that, by the assumption on $F$, one may chose the elements $a, b, c$ in such way that $-a b \in \sum F^{2}$, and that $\varphi^{\prime}$ then is also a torsion form.

The height of a quadratic form $\varphi$ over $F$, denoted by $h(\varphi)$, is by definition the height of its generic splitting tower (cf. [24, p. 160]). It can be given by the formula

$$
h(\varphi)=\left|\left\{i\left(\varphi_{K}\right) \mid K \in \mathcal{E}(F)\right\}\right|-1
$$

where $\mathcal{E}(F)$ is the set of finitely generated field extensions $K / F$. It is easy to see that

$$
h(\varphi) \leq \frac{1}{2}(\operatorname{diman}(\varphi)-\varepsilon(\varphi))
$$

This becomes an equality under certain conditions.

Lemma 7.5 Let $\varphi$ be a quadratic form over $F$. If $\operatorname{diman}(\varphi) \leq 5$, then

$$
h(\varphi)=\frac{1}{2}(\operatorname{diman}(\varphi)-\varepsilon(\varphi)) .
$$

Proof We may assume that $\varphi$ is anisotropic. For $\operatorname{diman}(\varphi) \leq 3$ the equality is obvious. Assume that $\varphi$ has dimension 4 or 5 . Let $K / F$ be a quadratic extension over which $\varphi$ becomes isotropic. If $i\left(\varphi_{K}\right)=1$, then $h(\varphi)=2$ and $\varphi \notin I^{2} F$, which yields the stated equality. It remains to consider the case where $i\left(\varphi_{K}\right)=2$. Then $\varphi$ contains a four-dimensional form 
$\pi$ over $F$ of trivial discriminant. If $\operatorname{dim}(\varphi)=4$ then $\varphi=\pi \in I^{2} F$, hence $h(\varphi)=1$ and $\varepsilon(\varphi)=2$, showing the claim. On the other hand, if $\operatorname{dim}(\varphi)=5$ then $\varphi$ is a neighbour of a threefold Pfister form $\rho$ over $F$. Let $L$ be the function field of $\varphi$ over $F$. With $\psi$ being the form over $F$ such that $\rho=\varphi \perp-\psi$ we obtain that $\psi_{L}$ is anisotropic, and further Witt equivalent to $\varphi_{L}$, because $\rho_{L}$ is hyperbolic. As $\operatorname{dim}(\psi)=3$ we obtain $i\left(\varphi_{L}\right)=1$ and conclude the claimed equality by using that it holds for $\psi_{L}$.

Theorem 7.6 Assume that $I_{t}^{3} F=0$. Let $\varphi$ be a form over $F$. Assume either that $F$ is an $E D$-field and $\left|\operatorname{sign}_{P}(\varphi)\right| \leq 5$ for all $P \in X(F)$, or that $\varphi$ is a torsion form. Then

$$
h(\varphi)=\frac{1}{2}(\operatorname{diman}(\varphi)-\varepsilon(\varphi)) .
$$

Proof We proceed by induction on $\operatorname{diman}(\varphi)$. For $\operatorname{diman}(\varphi) \leq 5$, the claim follows from (7.5). Assume that $\operatorname{diman}(\varphi) \geq 6$. Without loss of generality we assume that $\varphi$ is anisotropic. Any of the two alternative assumptions in the statement allows to conclude that $\varphi$ contains a torsion binary form $\beta$. We put $K=F(\sqrt{d})$ where $d$ is the discriminant of $\beta$. Then $K / F$ is a totally positive quadratic extension and $\varphi_{K}$ is isotropic. By (4.2) we have $i\left(\varphi_{K}\right)=1$, whence $\operatorname{diman}\left(\varphi_{K}\right)=\operatorname{dim}(\varphi)-2$. As $K / F$ is totally positive, the assumption $I_{t}^{3} F=0$ implies that $I_{t}^{3} K=0$. Moreover, if $F$ is an $E D$-field then so is $K$. By induction hypothesis we have $h\left(\varphi_{K}\right)=\frac{1}{2}\left(\operatorname{diman}\left(\varphi_{K}\right)-\varepsilon\left(\varphi_{K}\right)\right)$. Since $\operatorname{diman}(\varphi)=\operatorname{dim}(\varphi)=\operatorname{diman}\left(\varphi_{K}\right)+2$, we obtain

$$
\frac{1}{2}\left(\operatorname{diman}(\varphi)-\varepsilon\left(\varphi_{K}\right)\right)=h\left(\varphi_{K}\right)+1 \leq h(\varphi) \leq \frac{1}{2}(\operatorname{diman}(\varphi)-\varepsilon(\varphi)) .
$$

Thus, showing the equality $\varepsilon\left(\varphi_{K}\right)=\varepsilon(\varphi)$ would establish the claim.

Assume on the contrary that $\varepsilon\left(\varphi_{K}\right) \neq \varepsilon(\varphi)$. By the definition of the correction term $\varepsilon$ this means that we have $\varphi \in I F \backslash I^{2} F$ and $\varphi_{K} \in I^{2} K$. Let $\psi$ be the orthogonal complement of $\beta$ in $\varphi$ over $F$, whence $\varphi=\psi \perp \beta$. Since $K=F(\sqrt{d})$ with $d=d_{ \pm}(\beta)$, we conclude that $\psi \in I^{2} F$. Recall that $\beta$ is a torsion form. Hence, if $\varphi$ is torsion, then $\psi \in I_{t}^{2} F$, and by $(2.1, \mathrm{~b})$ this implies that $\psi$ is universal, contradicting the fact that $\varphi$ is anisotropic. Assume now that $F$ is an $E D$-field. We fix any element $a \in \Delta_{F}(\psi)$. Since $\beta$ is a torsion form over $F$, which is an $E D$-field, there is some $b \in \sum F^{2}$ such that $-a b \in \Delta_{F}(\beta)$. By $(2.1, a)$ then $b \psi=\psi$, so that $\varphi=b \psi \perp \beta$ is isotropic, again a contradiction.

Corollary 7.7 Assume that $I^{3} F(\sqrt{-1})=0$. Then for any torsion form $\varphi$ over $F$ one has

$$
h(\varphi)=t(\varphi)=t^{\prime}(\varphi)=\frac{1}{2}(\operatorname{diman}(\varphi)-\varepsilon(\varphi)) .
$$

Proof In view of (7.6) and (6.2), we only have to show that, for any non-hyperbolic torsion form $\varphi$ over $F$, one has $t^{\prime}(\varphi)=\frac{1}{2}(\operatorname{diman}(\varphi)-\varepsilon(\varphi))$.

It is not difficult to see that there exists a torsion form $\psi$ over $F$ with $\operatorname{dim}(\psi)=$ $2 t^{\prime}(\varphi)+\varepsilon(\varphi)$ and with the same discriminant and the same Clifford invariant as $\varphi$. Then $\varphi \perp-\psi \in I_{t}^{3} F=0$. Therefore we have $\operatorname{diman}(\varphi) \leq \operatorname{dim}(\psi)=2 t^{\prime}(\varphi)+\varepsilon(\varphi)$, i.e. $t^{\prime}(\varphi) \geq \frac{1}{2}(\operatorname{diman}(\varphi)-\varepsilon(\varphi))$. The other inequality follows from (7.4).

Acknowledgments The author expresses his gratitude to Detlev Hoffmann, David Leep, Jorge Morales, Claus Scheiderer, and Jean-Pierre Tignol for inspiring discussions and for their availability to his questions. He further gratefully acknowledges the financial support provided by the European RTN Network 'Algebraic $K$-Theory, Linear Algebraic Groups, and Related Structures' (HPRN-CT-2000-00287) and by the Swiss National Science Foundation (Grant No. 200020-100229/1). 


\section{References}

1. Amitsur, S.A., Rowen, L.H., Tignol, J.-P.: Division algebras of degree 4 and 8 with involution. Isr. J. Math. 33, 133-148 (1979)

2. Arason, J. Kr., Pfister, A.: Zur Theorie der quadratischen Formen über formalreellen Körpern. Math. Z. 153, 289-296 (1977)

3. Becher, K.J.: Minimal weakly isotropic forms. Math. Z. 252, 91-102 (2006)

4. Berhuy, G., Leep, D.B.: Divisible subgroups of Brauer groups and trace forms of central simple alge bras. Doc. Math. 6, 489-500 (2001)

5. Bourbaki, N.: Éléments de mathématique, XXIII. Première partie: Les structures fondamentales de l'analyse. Livre II: Algèbre. Modules et anneaux semi-simples, Chap. 8. In: Actualités Sci. Ind., vol 1261. Hermann, Paris (1958)

6. Elman, R., Lam, T.Y.: Quadratic forms and the $u$-invariant I. Math. Z. 131, 283-304 (1973)

7. Elman, R., Lam, T.Y.: Classification theorems for quadratic forms over fields. Comment. Math. Helv. 49, 373-381 (1974)

8. Hoffmann, D.W.: On the dimensions of anisotropic quadratic forms in $I^{4}$. Invent. Math. 131, 185-198 (1998)

9. Hoffmam, D.W.: Dimensions of anisotropic indefinite quadratic forms, I. Doc. Math., Quadratic Forms LSU Extra Volume, 183-200 (2001)

10. Jacob, B.: Indecomposable division algebras of prime exponent. J. Reine Angew. Math. 413, 181-197 (1991)

11. Kahn, B.: Quelques remarques sur le $u$-invariant. Sém. Théor. Nombres Bordeaux 2, 155-161 (1990). Erratum in: Sém. Théor. Nombres Bordeaux 3, 247 (1991)

12. Knebusch, M., Scheiderer, C.: Einführung in die reelle Algebra. In: Vieweg Studium: Aufbaukurs Mathematik, vol. 63. Vieweg, Braunschweig (1989)

13. Krüskemper, M.: On real local-global principles. Math. Z. 204, 145-151 (1990)

14. Lam, T.Y.: Introduction to quadratic forms over fields. In: Graduate Studies in Mathematics, vol. 67. American Mathematical Society, Providence (2005)

15. Marshall, M.: Some local-global principles for formally real fields. Canad. J. Math. 29, 606-614 (1977)

16. Marshall, M.: Review on 'J.-Ph. Monnier: On Lam's conjecture concerning signatures of quadratic forms. Arch. Math. (Basel) 75, 198-206 (2000).' Math. Reviews: MR1779862 (2001g:11056)

17. Merkurjev, A.S.; On the norm residue symbol of degree 2. (Russian) Dokl. Akad. Nauk. SSSR 261. 542-547 (1981). English translation: Soviet Math. Dokl. 24, 546-551 (1981)

18. Merkurjev, A.S.: Simple algebras and quadratic forms (Russian). Izv. Akad. Nauk SSSR Ser. Mat. 55, 218-224 (1991). Translation in Math. USSR-Izv. 38, 215-221 (1992)

19. Orlov, D., Vishik, A., Voevodsky, V.: An exact sequence for Milnor's K-theory with applications to quadratic forms. Preprint, 2000. (K-theory Preprint Archives: http://www.math.uiuc.edu/K-theory/0454/)

20. Pierce, R.S.: Associative algebras. In: Graduate Texts in Mathematics, vol. 88. Springer, New York (1982)

21. Prestel, A. (1984) Lectures on Formally Real Fields, LNM vol. 1093. Springer, Heidelberg

22. Prestel, A., Ware, R.: Almost isotropic forms. J. Lond. Math. Soc. Ser. II 19, 241-244 (1979)

23. Rowen, L.H.: Ring theory, vol. II. In: Pure and Applied Mathematics, vol. 128. Academic, Boston (1988)

24. Scharlau, W.: Quadratic and Hermitian forms. In: Grundlehren, vol. 270. Springer, Berlin (1985)

25. Voevodsky, V.: The Milnor Conjecture. Preprint 1996. (K-theory Preprint Archives: http:/www.math. uiuc.edu/K-theory/0170/) 